\title{
Drug coverage in treatment of malaria and the consequences for resistance evolution - evidence from the use of sulphadoxine/pyrimethamine
}

\author{
Allen L Malisa*1,2, Richard J Pearce ${ }^{3}$, Salim Abdulla2 , Hassan Mshinda2, Patrick S Kachur ${ }^{4}$ Peter Bloland ${ }^{4}$ and \\ Cally Roper ${ }^{3}$
}

\begin{abstract}
Background: It is argued that, the efficacy of anti-malarials could be prolonged through policy-mediated reductions in drug pressure, but gathering evidence of the relationship between policy, treatment practice, drug pressure and the evolution of resistance in the field is challenging. Mathematical models indicate that drug coverage is the primary determinant of drug pressure and the driving force behind the evolution of drug resistance. These models show that where the basis of resistance is multigenic, the effects of selection can be moderated by high recombination rates, which disrupt the associations between co-selected resistance genes.
\end{abstract}

Methods: To test these predictions, dhfr and dhps frequency changes were measured during 2000-2001 while SP was the second-line treatment and contrasted these with changes during 2001-2002 when SP was used for first-line therapy. Annual cross sectional community surveys carried out before, during and after the policy switch in 2001 were used to collect samples. Genetic analysis of SP resistance genes was carried out on 4,950 Plasmodium falciparum infections and the selection pressure under the two policies compared.

Results: The influence of policy on the parasite reservoir was profound. The frequency of $d h f$ r and $d h p s$ resistance alleles did not change significantly while SP was the recommended second-line treatment, but highly significant changes occurred during the subsequent year after the switch to first line SP. The frequency of the triple mutant $d h f r$ (N51I,C59R,S108N) allele (conferring pyrimethamine resistance) increased by 37\% - 63\% and the frequency of the double A437G, K540E mutant dhps allele (conferring sulphadoxine resistance) increased 200\%-300\%. A strong association between these unlinked alleles also emerged, confirming that they are co-selected by SP.

Conclusion: The national policy change brought about a shift in treatment practice and the resulting increase in coverage had a substantial impact on drug pressure. The selection applied by first-line use is strong enough to overcome recombination pressure and create significant linkage disequilibrium between the unlinked genetic determinants of pyrimethamine and sulphadoxine resistance, showing that recombination is no barrier to the emergence of resistance to combination treatments when they are used as the first-line malaria therapy.

\section{Background}

In a new era of anti-malarial treatment it is important to examine the use of past drugs and to determine which drug use practices are most likely to preserve drug efficacy and to examine their specific importance across a range of malaria endemicity settings. High recombination

\footnotetext{
*Correspondence: amalisa@suanet.ac.tz

1 Sokoine University of Agriculture, Department of Biological Sciences, Faculty of Science, SUA, PO Box 3038, Morogoro, Tanzania

Full list of author information is available at the end of the article
}

rates associated with high malaria transmission found in much of sub-Saharan Africa [1] have the potential to delay the emergence of resistance by disrupting associations between the multigenic components of resistance [2-4]. This consideration is important when resistance is multigenic, as is the case with chloroquine, quinine $[5,6]$ and SP [7], and will certainly be the case with the new artemisinin-based combination therapy (ACT). Mathematical models indicate that drug coverage is the primary determinant of drug pressure and the driving force 
behind the evolution of drug resistance [3,4]. These models show that where the basis of resistance is multigenic, the effects of selection can be moderated by high recombination rates, which disrupt the associations between co-selected resistance genes. In this study, the interplay between drug coverage, and the frequency of resistance genes in Plasmodium falciparum populations were examined in a highly endemic region of Africa [1]. Genetic changes occurring in the parasite reservoir of two rural sites in Tanzania were measured over a period of transition from low SP coverage while chloroquine was firstline treatment of uncomplicated malaria to higher coverage after SP became the first-line treatment.

The genetic determinants of pyrimethamine resistance are point mutations at codons 16, 50, 51, 59, 108 and 164 of the dhfr gene $[8,9]$. Genetic determinants of sulphadoxine resistance are mutations found at codons 436, 437, 540, 581 and 613 of the dhps gene [10,11]. In African $P$. falciparum, the presence of three dhfr mutations (N51I, C59R, S108N) together with two dhps mutations (A437G, $\mathrm{K} 540 \mathrm{E})$ prior to treatment is a significant predictor of SP treatment failure [12-14]. In southeast Africa, pyrimethamine resistance became established prior to sulphadoxine resistance. This was evidenced by in-vitro studies in Kenya, which showed that pyrimethamine resistance was common as early as 1988, while sulphadoxine resistance did not appear until 1993-1995 [15]. Molecular genetic studies have corroborated the in-vitro observations, showing that double mutant $d h f r$ was present in Kisumu, Kenya at least as early as 1981 [16], while resistant dhps only appeared between 1993 and 1995 [17]. The emergence of the dhps double mutant (A437G K540E) on a background of resistant $d h f r$ coincided with the emergence of clinically evident SP resistance in Africa. The dhps double mutant (A437G, K540E) was first recorded in Kenya between 1993 and 1995 [17], Tanzania in 1995 [18], and it was first seen in Malawi in 1995/ 1996 [19]. In north-eastern Sudan, it was absent in 1993 but had appeared by 1998 [20], and in KwaZulu Natal, South Africa, it was absent in 1995/1996 but had appeared by 1999 [21]. Analysis of microsatellite polymorphisms around resistant $d h f r$ and dhps suggests that underlying these events was a dispersal of the dhps double mutant allele on a background of resistant $d h f r$ throughout eastern and southern Africa [21].

Treatment of malaria in Tanzania is typically guided by official recommendations from the Ministry of Health and Social Welfare regarding drugs of choice for various situations. "First-line" treatment refers to the drug officially recommended as the drug of first choice for the treatment of uncomplicated malaria. "Second-line" treatment refers to the drug officially recommended as an alternative primarily to be used for treatment of patients in whom the first line treatment failed to clear the infec- tion and other select patients. "Third-line" treatment typically refers to the drug recommended for severely ill patients. In practice, few treatment failures are recognized and patients are often moved directly from first to third line treatment, consequently, little second line drug is used compared to the first line drug. SP replaced chloroquine as the recommended first line treatment on the Tanzanian mainland in August 2001. Prior to that SP was the recommended second line treatment. A survey of SP efficacy prior to national policy change showed a marked regional variability within Tanzania with clinical efficacy ranging between 76\% in Tanga and 93\% in Mwanza [22]. Tanga, in north-east Tanzania, was the first site in Africa where SP treatment failure was reported [23,24]. Our investigation took place in Kilombero/Ulanga and Rufiji Districts which are located $>400 \mathrm{~km}$ from Tanga and where resistance has been slower to appear. A study among children aged 6-59 months in Kilombero during 1999 found parasitological clearance was $94 \%$ over 28 days of follow-up after SP treatment [25].

To examine the genetic change at $d h f r$ and dhps loci under first and second line SP use in Kilombero/Ulanga and Rufiji Districts, malaria parasite samples were collected from residents of randomly selected households in the two districts, in a series of cross sectional surveys, over an area covering $29,400 \mathrm{~km}^{2}$ and including sites up to $300 \mathrm{~km}$ apart. Three cross sectional surveys were performed. The first survey occurred during July-September 2000, when CQ was first-line treatment and SP was second line. The second survey in July -September 2001 coincided with the implementation of a new national policy recommending use of SP as the first-line treatment of uncomplicated malaria. The third survey, in July-September 2002, occurred approximately one year after implementation of that policy change. In all, the surveys included 20,062 participants and yielded parasites from 4,950 malaria infections. By comparing genetic change between 2000 and 2001 with that which occurred between 2001 and 2002, the impact of the policy change on the genetic composition of the parasite population was quantified. Infections identified at community level include both symptomatic and asymptomatic participants. This method of sampling is preferable to sampling at a health clinic where by definition all infections are symptomatic, and many might have been pretreated. Since resistant parasites are selected by pre-treatment, the active surveillance approach yields a less biased sample of the circulating parasite population at community level.

\section{Methods}

Study area, subjects and samples

Community surveys were conducted during July, August and September of 2000, 2001 and 2002 in three rural dis- 
tricts of south-eastern Tanzania, Rufiji (Population = 170,000), Kilombero (Population $=220,000$ ) and Ulanga (Population $=160,000)$. The three districts were well matched in terms of predicted intensity and duration of malaria transmission and risk (MARA), relative access and overall utilization of health services (based on surveys), fairly usage of insecticide treated nets (ITNs) and relative proportion of urban peri-urban, rural population. The surveys were part of large combination therapy pilot implementation programme in Tanzania, the Interdisciplinary Monitoring Programme for Antimalarial Combination Therapy (IMPACT-TZ). IMPACT-Tanzania is a multiyear implementation research evaluation that rests on a collaborative platform incorporating the US Center for Disease Control and Prevention (CDC), the Ifakara Health Institute, London School of Hygiene and Tropical Medicine, and the Ministry of Health and Social Welfare including its National Malaria Control Programme, the Tanzania Essential Health Interventions Project and the Council Health Management Teams of Rufiji, Kilombero, Ulanga, Morogoro and Mvomero Districts. IMPACTTanzania is primarily supported by funding from the United States Agency for International Development, CDC and Wellcome Trust. For the purpose of the study, Kilombero and Ulanga Districts were treated as a single district because population movement between these two districts is high and the study population spans the border region. Plasmodium falciparum malaria transmission in the study area is intense (with an estimated entomological inoculation rate of 367 infectious bites per person per year [1]) and perennial with some seasonal fluctuation. A total of 20,062 adults and children belonging to randomly selected households participated in the study. A fingerprick blood sample for blood slide and filter paper bloodspot were collected from each individual in the household. The filter paper bloodspots were air-dried and stored at room temperature in self-sealing plastic bags with dessicant and stored dry at room temperature. All blood slide samples were screened by light microscopy for $P$. falciparum parasites. Bloodspots from microscopically positive subjects were selected and preserved at room temperature for molecular genotyping.

\section{Ethics}

Scientific and ethical clearance was granted from the Medical Research Council of the National Institute for Medical Research in Tanzania, the Centers for Disease Control and Prevention, USA, and the London School of Hygiene and Tropical Medicine. Written informed consent was obtained from all individuals or their guardians before collection of samples.

\section{DNA extraction}

The DNA was extracted from bloodspots dried on filter papers. A section of the dried blood spot filter paper was excised using a sterile blade or scissors, and soaked in a 1 $\mathrm{ml}, 0.5 \%$ saponin-1× phosphate buffered saline (PBS) overnight in a 96-deepwell plate. The section of filter paper was then washed twice in $1 \mathrm{ml}$ of $1 \times \mathrm{PBS}$ and finally, was boiled for $8 \mathrm{~min}$ in $100 \mu \mathrm{l}$ PCR quality water with $50 \mu \mathrm{l} 20 \%$ chelex suspension ( $\mathrm{pH} 9.5$ ).

\section{PCR amplification}

Nested PCR was used to amplify a 594 base pair (bp) fragment of $d h f r$ and a 711 bp fragment of dhps each containing the sequence where mutations are found. Primer sequences and PCR reaction conditions were previously described in [25]. PCR was performed in 96 well plates with $25-\mu$ l PCR reaction volumes containing final concentrations of $0.25 \mu \mathrm{M}$ oligonucleotide primers, $2 \mathrm{mM}$ $\mathrm{MgCl}_{2}, 250 \mu \mathrm{M}$ each deoxyribonucleotide triphosphate (dNTPs), and $1 \times$ Taq polymerase. $1 \mu$ l of DNA template was used in the outer (primary) PCR reaction mixture for $d h f r$ and dhps amplifications. For the inner (secondary) dhps reactions $1 \mu \mathrm{l}$ of the outer PCR product was used. The outer $d h f r$ PCR products were diluted three fold before $1 \mu \mathrm{l}$ was introduced into the inner PCR reaction mixtures.

\section{Molecular genotyping of point mutations by sequence specific oligonucleotide probing (SSOP)}

The amplified PCR products were screened for $d h f r$ and dhps sequence variants at 10 loci where single nucleotide polymorphisms (SNPs) related to anti-malarial drug resistance are known to occur. The sequence changes (and the amino acid substitutions they code for) are summarized in Table 1.

PCR products were spotted in a 12 by 8 -grid and cross linked onto nylon membranes and probed for sequence polymorphisms by hybridization to specific oligonucleotide probes described previously [26]. For analysis of samples collected in 2000, the visualization of hybridized digoxygenin labelled probes on membranes was performed by the alkaline phosphatase-catalysed breakdown of the CSPD substrate (Roche Boehringer Mannheim, Mannheim, Germany) and visualized by exposure on Hyperfilm-ECL (Amersham Pharmacia Biotech, Little Chalfont, Buckinghamshire, United Kingdom), according to Boehringer Mannheim recommendations and previously described [27]. For analysis of samples collected in 2001 and 2002 the probed blots were visualized using ECF substrate and detection using a phosphoimager $\left(\mathrm{STORM}^{\circ}\right)$. Inspection of autoradiographic films was carried out by light box illumination, while the phophoimager output was recorded through viewing of digitallycaptured images of chemifluorescent signal. The change in the method by which probe hybridization signal was visualized did not affect the results in any way since the probes and hybridization conditions were unchanged. 
Table 1: The nucleotide and amino acid substitutions at (a) dhfr and (b) dhps genes screened for by PCR-SSOP

\begin{tabular}{|c|c|c|c|c|c|}
\hline dhfr & & & & & \\
\hline Codon & 50 & 51 & 59 & 108 & 164 \\
\hline \multirow[t]{2}{*}{ Wild type } & Cys (C) TGT & Asn (N) AAT & Cys (C) TGT & Ser (S) AGC & Ile (I) ATA \\
\hline & & AAC & & & \\
\hline \multirow[t]{4}{*}{ Mutant } & $\operatorname{Arg}(R)$ & Ile (I) & $\operatorname{Arg}(R)$ & Asn (N) & Leu (L) \\
\hline & CGT & ATT & CGT & AAC & TTA \\
\hline & & & & Thr (T) & \\
\hline & & & & ACC & \\
\hline \multicolumn{6}{|l|}{ dhps } \\
\hline Codon & 436 & 437 & 540 & 581 & 613 \\
\hline \multirow[t]{2}{*}{ Wild type } & Ser $(S)$ & Ala (A) & Lys (K) & Ala (A) & Ala (A) \\
\hline & TCT & $\mathrm{GCT}$ & AAA & GCG & GCC \\
\hline \multirow[t]{6}{*}{ Mutant } & Phe (F) & Gly (G) & Glu (E) & Gly (G) & Ser (S) \\
\hline & TTT & GGT & GAA & GGG & TCC \\
\hline & Ala (A) & & & & Thr (T) \\
\hline & GCT & & & & ACC \\
\hline & Cys (C) & & & & \\
\hline & TGT & & & & \\
\hline
\end{tabular}

The stringency and specificity of the hybridization process was confirmed by inspection of a series of 4 controls with a known single genotype variant sequence. All blots with non-specifically bound probes were stripped and reprobed. A SNP was considered to be present in the PCR product when the intensity of signal was higher than that of the background. The blots were scored independently by two people.

In this analysis, the aim was to establish the relative abundance of different point mutation haplotypes at $d h f r$ and dhps. Since bloodstage P. falciparum is haploid, this is very straightforward when an infection consists of a single genotype because only one form of sequence at every SNP locus is seen. When infections are composed of multiple genotypes a mixture of different sequence variants occurs making the inference of point mutation haplotypes within that infection more difficult.

The presence, absence, and relative abundance of hybridization signal for every probe were recorded at each locus. A sample was considered to have a single haplotype when only one sequence variant was found at each locus. Blood samples were categorized as having a single, a majority, or a mixture of sequence at each SNP locus. Majority and mixed genotype infections were differentiated according to the relative intensity of signal. If the hybridization signal of the minority sequence was greater than half the intensity of the majority then an infection was classified as mixed. To determine the relative abundance of different point mutation haplotypes in the parasite population, one haplotype only was counted from each infection and those mixed infections where haplotypes could not be resolved were omitted from the calculation of haplotype frequencies. Hence, frequency data is based upon a subset of isolates which were either unmixed or had a predominating majority haplotype. A breakdown of the proportions of isolates which successfully PCR amplified and which were genotyped as single, majority or mixed haplotype infections is given in Table 2.

\section{Statistical analysis}

Statistical comparison of allele frequencies at $d h f r$ and dhps in the various sites was carried out using chisquared analysis in STATA version 9.2 [28]. The calcula- 
tion of binomial exact 95\% confidence intervals was carried out using STATA version 9.2. Linkage disequilibrium analysis was performed using Arlequin software.

\section{Role of the funding source}

This study was funded through an interagency agreement between the United States Agency for International Development (USAID) and CDC and a cooperative agreement between $\mathrm{CDC}$ and the Ifakara Health Institute (IHI). USAID did not participate in the design, collection, analysis, or interpretation of the data, in the writing of the report, or in the decision to submit for publication.

\section{Results}

Of 20,062 people sampled, 4,950 were identified as infected with P. falciparum. DNA was extracted from the 4,950 P. falciparum positive bloodspots and PCR amplification of dhfr and dhps performed once, giving a combined rate of PCR amplification success of $69 \%$ for both genes (Table 2). The amplified products were screened for all the variant sequences described in Table 1. Out of the 3,436 isolates, which amplified successfully for $d h f r$,

\section{Allelic haplotypes at $d h f r$ and dhps genes}

The point mutations found in the $d h f r$ gene were N51I, C59R and S108N. They were found in the following haplotypic arrangements NCS, NCN ,NRN, ICN and IRN which are common throughout East Africa and have been previously reported in Tanzania [26], Malawi [29], Kenya $[12,30]$, and Uganda [31,32]. Two rare combinations of mutations were found; N51I with C59R (IRS) was found in a single individual (previously reported in Uganda [31], and C59R alone (NRS) was found in one sample only and has not been previously reported, (again sequence confirmation of these rare haplotypes needs to be done).

Five dhps mutations were found (S436A, S436F, S436C, $\mathrm{A} 437 \mathrm{G}$, and $\mathrm{K} 540 \mathrm{E}$ ) in nine distinct haplotypic arrangements; five of which (SAK, AAK, SGE ,SGK, and SAE ) have been described previously in isolates from East Africa [12,26,30-32] while the remaining four (CAK, FAK, AAE , and FAE) were found in extremely low frequency and have not been reported before, presumably because of their rarity, although sequencing confirmation is also needed to verify their existence.

The change in frequency of the $d h f r$ IRN and the $d h p s$

Table 2: Annual survey 2000-2002, malaria positive samples and their PCR outcome in Rufiji and Kilombero/Ulanga populations.

\begin{tabular}{|c|c|c|c|c|c|c|}
\hline \multirow[b]{2}{*}{ Year } & \multicolumn{3}{|c|}{ Rufiji } & \multicolumn{3}{|c|}{ Kilombero Ulanga } \\
\hline & 2000 & 2001 & 2002 & 2000 & 2001 & 2002 \\
\hline Survey population & 2844 & 3285 & 3349 & 3289 & 3197 & 4098 \\
\hline P. falciparum positive & 778 & 908 & 854 & 955 & 580 & 875 \\
\hline PCR amplified $d h f r$ & 549 & 683 & 687 & 404 & 488 & 686 \\
\hline PCR amplified $d h p s$ & 521 & 592 & 725 & 444 & 347 & 720 \\
\hline \multirow[t]{2}{*}{ Single or majority $d h f r$} & 455 & 420 & 527 & 376 & 238 & 489 \\
\hline & $83 \%$ & $62 \%$ & $77 \%$ & $93 \%$ & $49 \%$ & $71 \%$ \\
\hline \multirow[t]{2}{*}{ Single or majority dhps } & 417 & 519 & 596 & 365 & 294 & 603 \\
\hline & $80 \%$ & $88 \%$ & $82 \%$ & $82 \%$ & $85 \%$ & $84 \%$ \\
\hline \multirow[t]{2}{*}{ Single or majority $d h f r+d h p s$} & 288 & 278 & 404 & 190 & 138 & 381 \\
\hline & $55 \%$ & $47 \%$ & $59 \%$ & $47 \%$ & $40 \%$ & $55 \%$ \\
\hline
\end{tabular}

$71 \%$ were single or majority genotype infections and the point mutation haplotypes could easily be resolved. Of the 3,412 samples, which amplified successfully for dhps, $81 \%$ were single or majority genotype with resolvable haplotypes.
SGE haplotypes, which have the greatest significance for SP efficacy are shown in Figures $1 \mathrm{a}$ and $1 \mathrm{~b}$. Changes occurring under the CQ policy (2000 to 2001) are markedly different to those occurring under the SP policy (2001-2002). The frequency of the dhps double mutant 
A437G K540E, shown in Figure 1a, did not change significantly between 2000 and 2001 in either Rufiji (0.08-0.09) ( $\mathrm{p}>0.411,95 \% \mathrm{CI})$ or in Kilombero/Ulanga (0.13-0.11) (p $>0.497,95 \% \mathrm{CI}$ ). Contrastingly, there was a highly significant change between 2001 and 2002 in both Rufiji (0.090.25 ) (2.5 fold increase $\mathrm{p} \leq 0.0001,95 \% \mathrm{CI}$ ) and Kilombero/Ulanga (0.11-0.27) (2.6 fold increase $\mathrm{p} \leq 0.0001)$. The frequency of the dhps double mutant haplotype was remarkably similar in Kilombero/Ulanga and Rufiji and there were no significant differences between the two sampling sites at any time point.

The change in frequency of the $d h f r$ IRN and the $d h p s$ SGE haplotypes, which have the greatest significance for SP efficacy are shown in Figures $1 \mathrm{a}$ and $1 \mathrm{~b}$. Changes occurring under the CQ policy (2000 to 2001) are markedly different to those occurring under the SP policy (2001-2002). The frequency of the dhps double mutant A437G K540E, shown in Figure 1a, did not change significantly between 2000 and 2001 in either Rufiji (0.08-0.09) ( $\mathrm{p}>0.411,95 \% \mathrm{CI})$ or in Kilombero/Ulanga (0.13-0.11) (p $>0.497,95 \% \mathrm{CI}$ ). Contrastingly, there was a highly significant change between 2001 and 2002 in both Rufiji (0.090.25 ) (2.5 fold increase $\mathrm{p} \leq 0.0001,95 \% \mathrm{CI}$ ) and Kilombero/Ulanga (0.11-0.27) (2.6 fold increase $\mathrm{p} \leq 0.0001)$. The frequency of the dhps double mutant haplotype was remarkably similar in Kilombero/Ulanga and Rufiji and there were no significant differences between the two sampling sites at any time point.

Changes in the frequency of the $d h f r$ triple mutant allele IRN are shown in Figure 1b. The frequency was significantly higher in Rufiji than in Kilombero/Ulanga at all time points. Between 2000 and 2001, there was an increase in frequency in both Kilombero/Ulanga (0.280.31 ) (p > 0.400, 95\% CI) and Rufiji (0.38-0.47) ( $<<0.007$, 95\% CI), yet between 2001 and 2002 the increase was greater and more highly significant in both Kilombero/ Ulanga (0.31-0.52) (p $\leq 0.0001,95 \% \mathrm{CI})$ and Rufiji (0.47$0.63)(\mathrm{p} \leq 0.0001,95 \% \mathrm{CI})$.

In a further subset of samples where both $d h f r$ and $d h p s$ sequences were unmixed, it was possible to measure the frequency of two locus genotypes. In figure 1c, the frequency of the triple $d h f r+$ double dhps genotype in the two populations is compared. The initial frequency was around 0.05 in both the districts and there was no change between 2000 and 2001 in Kilombero/Ulanga ( $p>0.824$, 95\% CI) or Rufiji (p > 0.186, 95\% CI) but a remarkable 4 fold increase to frequencies of 0.21 and 0.24 occurred between 2001 and 2002 in Kilombero/Ulanga ( $<<0.0001$, 95\% CI) and Rufiji (p < 0.0001, 95\% CI).

\section{Haplotypes conferring intermediate levels of resistance}

The effect of changing policy on the frequency of sensitive and double mutant $d h f r$ alleles is shown in Figure 2. The increase of the triple mutant allele acted to displace

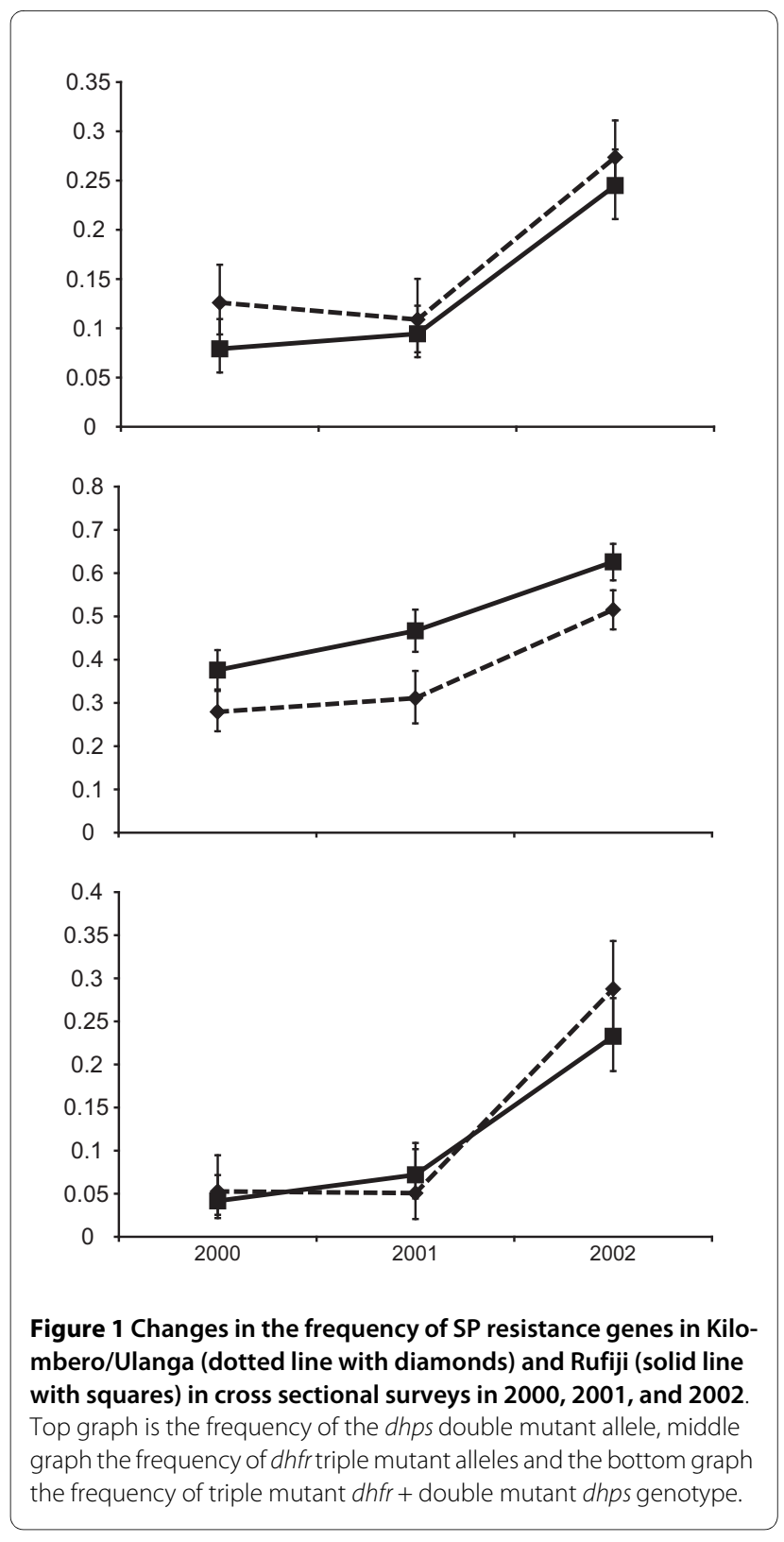

sensitive alleles, which show a substantial decline in Kilombero/Ulanga 2000-2001 (0.51-0.47) ( p > 0.352, 95\% CI), 2001-2002 (0.47-0.28) ( $\mathrm{p} \leq 0.0001,95 \% \mathrm{CI})$ and Rufiji 2000-2001 (0.36-0.30) (p > 0.092, 95\% CI) and 2001-2002 (0.30-0.18) ( $\mathrm{p} \leq 0.0001,95 \% \mathrm{CI})$. The double mutant $d h f r$ alleles, which confer intermediate levels of resistance invitro [33], neither increased nor decreased, remaining at a frequency of around 10\% in both sites throughout all surveys.

As well as the highly resistant dhps A437G K540E double mutant, a number of single 436 mutant alleles were recorded. Among these, by far the most common was the S436A, which was consistently found at frequencies of $10 \%-20 \%$ at all time points in both districts. Figure 3 
shows the frequencies of the sensitive S436A single mutant and the A437G K540E double mutant alleles through time in both districts. From these data it is clear that the rising frequency of the double mutant allele displaced the sensitive allele, which decreased significantly during 2001-2002 in both Kilombero/Ulanga (0.7-0.6) (p $\leq 0.001,95 \% \mathrm{CI}$ ) and Rufiji (0.77-0.60) ( $\mathrm{p} \leq 0.0001,95 \%$ $\mathrm{CI})$. Interestingly, the frequency of the A436A allele remained static at all time points in both sites.

\section{Linkage disequilibrium}

To examine the effect of simultaneous selection by pyrimethamine on $d h f r$ and sulphadoxine on dhps, we looked at two-locus genotypes sampled from both sites in the three successive surveys. Taking the subset of samples for which point mutation haplotypes could be unequivocally resolved for both genes, we compared the observed with expected frequencies generated from contingency tables (given in full in Additional file 1). No significant linkage disequilibrium was found between $d h f r$ and dhps loci in either 2000 or 2001. Since these surveys were both conducted while SP was second-line therapy, the data was pooled and, in Figure 4, linkage disequilibrium (d') is
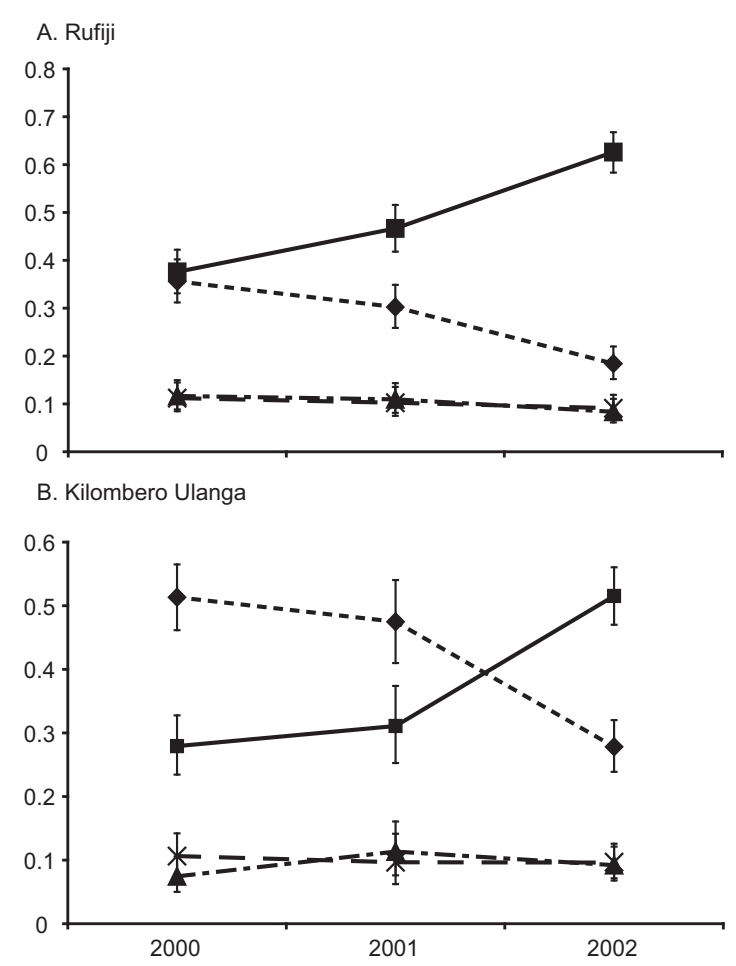

Figure 2 Allele frequency changes at dhfr in Rufiji (A), and Kilombero/Ulanga (B). The sensitive allele (dotted line with diamond), the triple mutant N51I+C59R + S108N allele (solid line with squares), and double mutant C59R + S108N (dash-dot line with triangles), double mutant N511 + S108N(dashed line with X).

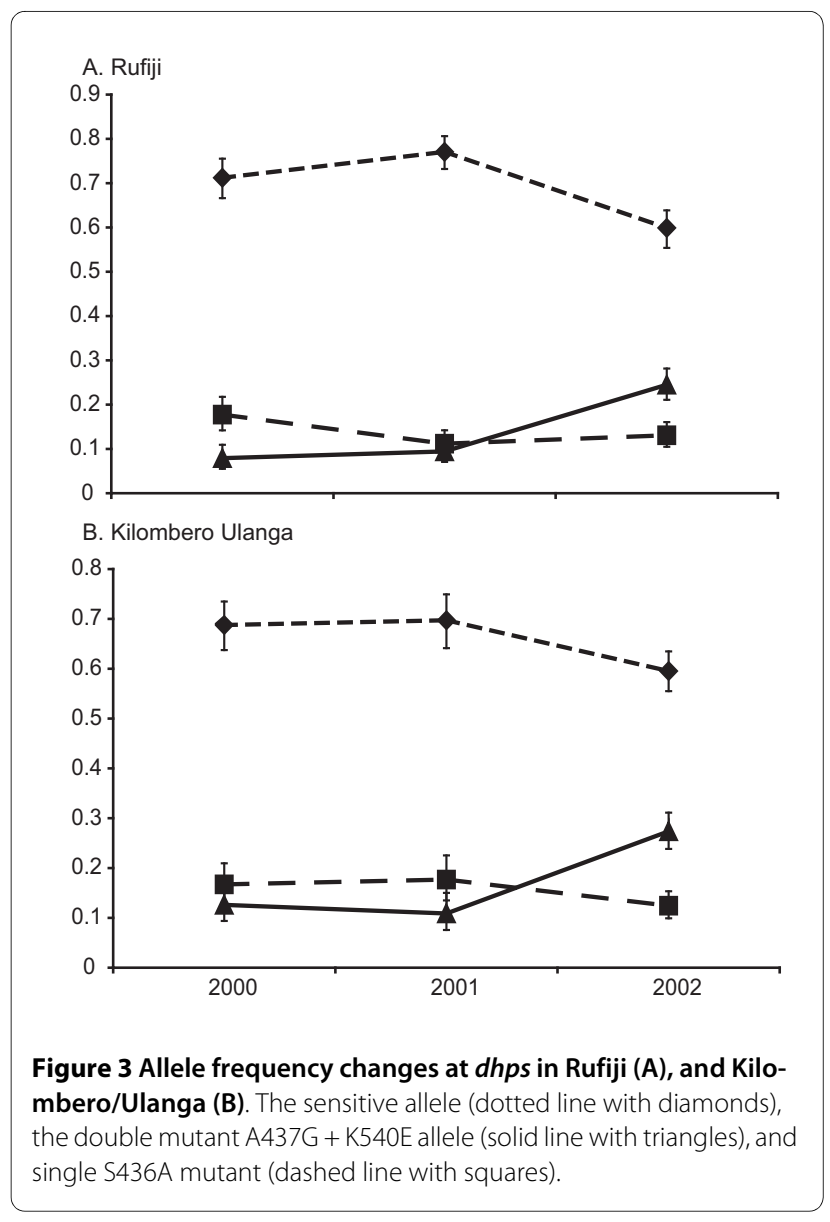

compared under second line SP selection (2000 and 2001 combined) and during first line SP selection (in the 2002 survey). There was a significant association between the $d h f r$ triple mutant allele and the dhps double mutant allele after the change in policy. In Kilombero/Ulanga during 2000-2001, 17 of the 328 unmixed samples carried the $d h f r$ triple $d h p s$ double mutant genotype $\left(\mathrm{d}^{\prime}=0.1015\right.$, p > 0.2885, 95\% CI) while in Rufiji 2000-2001, 32 of 566 unmixed samples had the $d h f r$ triple dhps double mutant genotype $\left(d^{\prime}=0.1821, p>0.0760,95 \%\right.$ CI). By 2002 there was a highly significant association between these alleles. In Kilombero/Ulanga 84 of 381 unmixed samples carried the dhfr triple dhps double mutant genotype and 64 were 'expected' (d' = 0.3633, $\mathrm{p} \leq 0.0001,95 \% \mathrm{CI}$ ) and in Rufiji 94 of 404 unmixed samples carried this same genotype compared to 79 'expected' (d' $=0.3184, \mathrm{p} \leq 0.001,95 \% \mathrm{CI})$. The $\mathrm{d}^{\prime}$ index of linkage disequilibrium, between the dhps A437G + K540E double mutant and dhfr double mutant allele was not significantly changed (Figure 4B) but a negative association between the dhps double mutant allele and $d h f r$ sensitives emerged under first line SP treatment (Figure 4C) (Rufiji $\mathrm{p} \leq 0.0001,95 \% \mathrm{CI}$, and Kilombero/ Ulanga $\mathrm{p} \leq 0.0001,95 \% \mathrm{CI}$ ). 

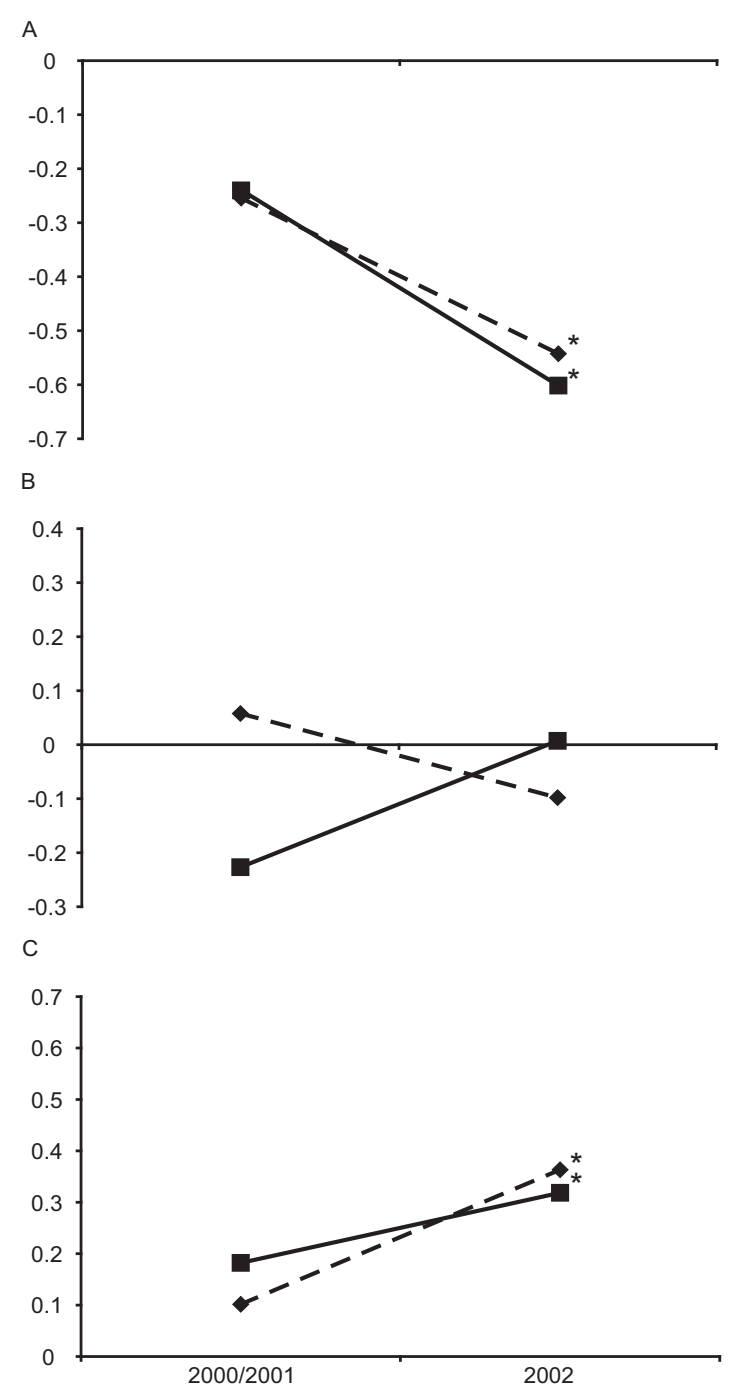

Figure 4 Linkage disequilibrium between the dhps A437G + K540E allele and different alleles at $d h f r(\mathrm{~A})$ the $d h f r$ triple mutant $\mathrm{N} 511+\mathrm{C} 59 \mathrm{R}+\mathrm{S} 108 \mathrm{~N}$ (B) dhfr double mutants C59R + S108N and $\mathbf{N} 511+\mathbf{S 1 0 8 N}$ (C) the dhfr sensitive allele. The d'values for $2000+$ 2001 (Kilombero/Ulanga $n=328$, Rufiji $n=566$ ) combined and for 2002 (Kilombero/Ulanga $n=381$, Rufiji $n=404$ ) are shown. Significant deviation between observed from expected occurred in 2002 indicated by $*(p<$ or $=0.001)$.

\section{Measurement of haplotype frequencies}

Measurement of haplotype frequencies is complicated by the existence of mixed infections. If multiple haplotypes are counted from mixed infections the frequency of rare allelic haplotypes is overestimated. Haplotype frequency measures presented were based solely on infections where a single or majority haplotype was detected, and only one haplotype was scored per individual infection, while mixed infections were excluded because point mutation haplotypes could not be determined. The over- all proportion of mixed infections excluded from the frequency estimations was $29 \%$ of infections for $d h f r$ and $19 \%$ of infections for dhps.

To examine the extent of the underlying rate of mixture in infections and test the robustness of this frequency estimates, this study looked at three unlinked microsatellite markers (Poly A, Pfpk2 and TA109) in 178 and 180 samples from 2002 survey from Kilombero/Ulanga and Rufiji, respectively. The minimum number of co-infecting genotypes in each infection was determined as the greatest number of alleles at any of the three microsatellite loci. This number is often referred to as the "multiplicity of infection" or (MOI). In Figure 5 the distribution of MOI in the two districts is compared showing that they are similar. The presence/absence of dhps A437G and $\mathrm{K} 540 \mathrm{E}$ among the same infections was used to test whether the distribution of the dhps double mutant alleles was consistent with expectation using a method devised by Schneider et al [34] (see Additional file 1). This uses maximum likelihood to predict the underlying frequency of resistance alleles based on measures of the multiplicity of infection and the presence/absence of resistance mutations in those infections. The analysis predicted an underlying frequency of the dhps double mutant of 0.276 in Rufiji (where the frequency among single and majority genotype infections was 0.274 ) and a frequency of 0.262 in Kilombero Ulanga (where the frequency among single and majority genotype infections was 0.245$)$. The estimates using these two approaches are very consistent showing that no obvious bias is introduced into the estimation of frequencies by excluding mixed infections.

The robustness of this approach for estimating allele frequencies in populations sampled by blood survey has been demonstrated in a study by Anderson et al [35] comparing three different methods. All three methods gave equivalent frequency values. The predominant allele

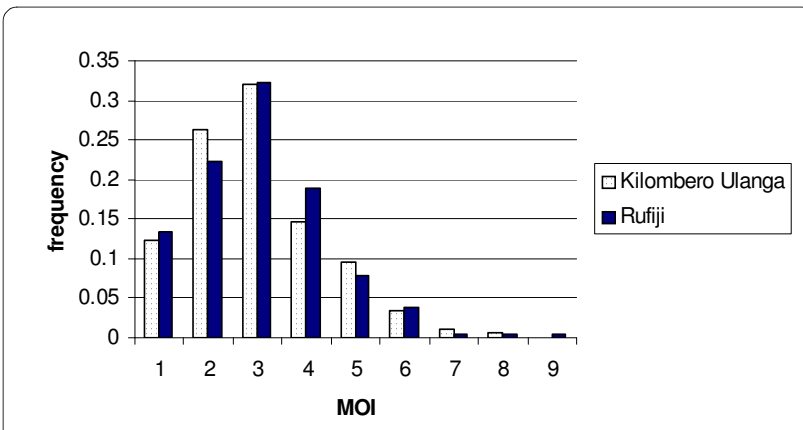

Figure 5 The minimum number of co-infecting genotypes (multiplicity of infection or MOl) was determined by measuring the number of alleles in every sample at 3 unlinked microsatellite loci (Poly A, Pfpk2 and TA109). Here the MOI in 178 samples from Kilombero/Ulanga 2002 (white) and 180 samples Rufiji 2002 (black) are compared. 
adopted in our study is favoured because it utilizes data from as many individuals as possible while still giving reliable haplotype data. Importantly the MoI did not vary significantly between treatments at any time it therefore cannot be the source of any systematic bias in our analysis. Majority or minority, the genotypes important in the spread of resistance must, by definition, appear in greater frequency in subsequent surveys. The power of examining a series of cross sectional surveys is its capacity to show the significant micro-evolutionary genetic changes over time.

\section{Discussion}

\section{Policy and genetic change}

The influence of policy change on the genetic composition of the parasite population was profound. While the frequency of $d h f r$ and dhps alleles did not change significantly during 12 months of first line CQ treatment and second line SP, the switch to a policy of first line SP caused the frequency of the triple mutant $d h f r$ allele to increase by $37 \%$ to $63 \%$ and the frequency of the double mutant dhps allele to increase $200-300 \%$. A combination of these two alleles is predictive of SP treatment failure in-vivo [12-14] and the rapid increase in the frequency of this genotype from $5.5 \%$ to $22.7 \%$ between 2001 and 2002 is consistent with findings of a similar study in Mozambique [36], yet suggests that the outlook for SP efficacy in subsequent years would be increasingly bad. In fact, this observation is borne out by the results of SP treatment efficacy monitoring in south-east Tanzania during 2003 which found that $49 \%$ of SP treatments failed by day 28 [37]. A national policy decision was made in 2006 to switch the recommended first-line treatment to artemether plus lumefantrine combination treatment.

Household interviews conducted simultaneously showed that there was a sharp increase in SP use after the policy transition (a 5-6 fold increase between 2000 and 2002). Prior to the introduction of SP as first line therapy SP was widely available for self-treatment via purchase from shops [38], as well as through formal health facilities as a second-line treatment. Although SP is currently being replaced as the recommended first-line treatment for malaria in Tanzania and much of the rest of Africa, it will continue to be used in intermittent preventive treatment of infants and pregnant women. The drug pressure applied when SP use is restricted to intermittent programmes of treatment in infants and pregnant women will more closely resemble the situation of 2000-2001. The predicted consequences of more restricted use of SP are, first, that the rate at which frequency of resistance alleles increase should decline, and second, that the association between the triple mutant $d h f r$ allele and double mutant dhps allele maintained by high coverage drug selection will diminish. Both these predictions pre-sup- pose that the resistance alleles had not already become fixed in the population.

\section{The spread of resistance}

For successful spread, a resistance mutant must be transmitted at a faster rate than the sensitive form. This occurs in the presence of drug pressure because of the differential survival and reproduction rates conferred by these alleles. The population-wide rates of change observed in the present study are attributable to changes in SP coverage during that period. In order to quantify drug pressure under a specific policy intervention, its effect upon the relative abundance, or frequency, of every haplotype needs to be measured because each allele is subject to a differing selection pressure by virtue of its differing resistance properties.

The finding that first line use of SP brought about the same genetic changes independently in Kilombero/ Ulanga and in Rufiji indicates they are likely to have broad applicability elsewhere. But direct transposition to predict rates in other settings should take into account differences in access to drugs and in the infrastructure for provision of healthcare services that can differ substantially from country to country. Importantly, from a molecular genetic perspective, recent studies in west and central African countries still show the dhps A437G, $\mathrm{K} 540 \mathrm{E}$ double mutant allele to be absent or very rare $[39,33,40]$. Only once these alleles are established will the rates of change observed here be predictive of change in such populations.

\section{Evolutionary history of resistance alleles}

By following haplotype frequencies over time, the contrasting behaviors of mutant alleles which confer high, intermediate or mild resistance were described. The frequency of weakly resistant alleles such as the $d h f r$ double mutant and the dhps single mutant did not respond to the increase in SP use. During the course of the three annual surveys, both $d h f r$ double mutant alleles maintained a steady frequency of around 10\%, and the dhps single mutant maintained a frequency of around 15\%. They were neither displaced by the highly resistant alleles, as the sensitive alleles were, nor did they increase in frequency in response to elevated drug pressure.

Double mutant $d h f r$ alleles are often regarded as precursors of the triple mutant since they can potentially convert to the highly resistant triple mutant allele by the simple acquisition of a single point mutation. Somewhat surprisingly, flanking microsatellite analysis has shown that the triple mutants in southeast Africa [21], and in the Kilombero/Ulanga site specifically [41], belong to a single lineage which originated in Asia [42]. The double mutant $d h f r$ alleles found in this site belong to a restricted number of independently derived lineages [21], which are in 
all probability of African origin. So while the increase in frequency of triple mutant allele in Kilombero/Ulanga and Rufiji can be explained by the expansion of the Asian derived lineage under selection by SP treatment, the steady persistence of the mildly resistant double mutant dhfr lineages at around 10\% requires some further explanation. The existence of conserved flanking region around them is evidence of selection [21] and there is further evidence that double mutant $d h f r$ alleles can gain fitness advantage in specific circumstances. For example, they have been associated with treatment failure in individuals who have not had sufficient exposure to malaria to have acquired immunity $[14,43]$ and while not affecting clinical efficacy in Colombia, the N51I + S108N double mutant was associated with prolonged parasite clearance times and with gametocytaemia 14 and 28 days after treatment, indicating they confer survival and reproductive advantages [44]. It has also been proposed that exposure to sub-therapeutic drug levels might select for weakly resistant, or drug tolerant parasites which are newly inoculated into people who recently received treatment [45].

An alternative explanation for the continuing existence of $d h f r$ double mutant alleles is that they pre-date the use of SP. Pyrimethamine was used as monotherapy during the 1950s and 60s and numerous studies showed that resistance quickly arose locally in response to drug pressure [46-48]. Double mutant $d h f r$ alleles could have been the basis of these early reports of pyrimethamine resistant malaria and may well have preceded the arrival of the dhfr triple mutant. Supporting evidence comes from studies in Kenya where double mutant alleles were in the majority in a panel of isolates collected as early as 19N51I + S108N; 21\% and C59R + S108N; 47\% (16). These reports are concurrent with measures of intermediate resistance of around 20\% in Kilifi, coastal Kenya between 1984 and 1989. This rose to 92\% between 1993 and 1995 [49], by which time the triple mutant was becoming more prevalent in Kilifi [50].

\section{The evolution of multi-drug resistance}

By combining $d h f r$ and $d h p s$ data the frequency of the highly resistant $d h f r$-dhps genotype in each of three time points was estimated. The frequency of this genotype more than quadrupled between 2001 and 2002 in Kilombero/Ulanga and in Rufiji, and it also was found greatly in excess of the numbers expected on the basis of random association. Such population-wide associations between unlinked resistance genes are expected to occur when two drugs with independent modes of action are combined, or where resistance to a single drug is controlled by unlinked mutations. The association occurs because of the combined effects of two selection processes which occur simultaneously. The fully resistant parasites survive better than genotypes with partial resistance. In addition, the drug treatment itself promotes assortative mating among resistant survivors of treatment by purging the coinfecting sensitive genotypes. Recombination, which occurs between gametocytes taken up in the same blood meal, is a force which can disrupt these associations, particularly in high transmission settings and it has been proposed that high recombination might slow the spread of resistance in such areas [51,52]. Models have indicated that recombination can counteract the effect of drug pressure most effectively when the resistance alleles involved are rare, or when drug pressure is low $[4,52,53]$. The evidence of this study endorses this conclusion since the population-wide association of $d h f r$ triple and $d h p s$ double mutant alleles was not significant when drug pressure was weak but became apparent immediately drug pressure increased. This is a significant consideration in the era of combination treatments for malaria. It suggests that in high transmission settings there may be value in using a suite of anti-malarial drugs for different treatment applications such as intermittent preventive treatment in pregnancy or intermittent preventive treatment of infants rather than blanket use of the same regimen across all malaria treatment applications.

It is widely agreed that malaria treatments should be used in a manner which will minimize drug pressure and limit the growth of resistance. The difficulty lies in gathering empirical support for specific policy alternatives in order to achieve this. Measurements from a variety of different endemic contexts are needed in order to evaluate the effects of policy and drug resistance and the body of evidence from the field is growing [54]. A key observation here is that when resistance alleles are present at low frequency and selection is applied they then spread rapidly. Clearly the first emphasis of policy should always be to minimize the emergence of new mutations and this might be achieved through use of combination therapy. Since this study observed a strong link between government guidelines on treatment practice and drug pressure this is encouraging to the view strategic policy on treatment has the potential to be used in prolonging the useful life of drugs in rural Africa.

\section{Additional material}

Additional file 1 Supplementary information. Observed and expected values for linkage disequilibrium analyses and complete data of haplotype frequencies detected in the study

Competing interests

The authors declare that they have no competing interests.

\section{Authors' contributions}

ALM: Participated in study design, carried out the molecular genotyping, statistical analysis, interpretation of the data and drafting of the manuscript. RP: Participated in molecular genotyping, statistical analysis, interpretation of the data 
and critical review of the manuscript for important intellectual content. SA and PK: Oversaw all aspects of the study, including design and execution of the field work, analysis and interpretation of the data and critical review of the manuscript for important intellectual content. HM: Participated in the conception and designing of the study and critical review of the manuscript for important intellectual content. PB: Original conception and designing of the study and critical review of the manuscript for important intellectual content. CR: Conception of the study, oversaw all the molecular aspect of the study, participated statistical analysis, interpretation of the data and drafting of the manuscript. All the authors read and approve final manuscript for submission

\section{Acknowledgements}

The authors are grateful to all people who participated in the prevalence surveys in Kilombero, Ulanga and Rufiji districts. The Interdisciplinary Monitoring Project for Antimalarial Combination Therapy in Tanzania (IMPACT-Tz) is funded by USAID, CDC, and Wellcome Trust. The co-Principal Investigators are Salim Abdulla and Peter Bloland. Cally Roper and Richard Pearce are supported by a Wellcome Trust Fellowship (ref 060714) awarded to CR. The authors also thank the staff who carried out the cross sectional surveys and the support staff of $\mathrm{IH}$.

\section{Author Details}

'Sokoine University of Agriculture, Department of Biological Sciences, Faculty of Science, SUA, PO Box 3038, Morogoro, Tanzania, 2lfakara Health Institute (IHI), PO Box 53, Ifakara, Kilombero District, Tanzania, ${ }^{3}$ London School of Hygiene and Tropical Medicine, Pathogen Molecular Biology Unit, Department of Infectious Tropical Diseases, Keppel Street, London, WC1E 7HT, UK and 4Malaria Branch, Division of Parasitic Diseases, National Center for Infectious Diseases, Centers for Disease Control and Prevention (CDC), Atlanta, GA, USA

Received: 19 March 2010 Accepted: 5 July 2010

Published: 5 July 2010

\section{References}

1. Hay SI, Rogers DJ, Toomer JF, Snow RW: Annual Plasmodium falciparum entomological inoculation rates (EIR) across Africa: literature survey, Internet access and review. Trans R Soc Trop Med Hyg 2001, 94:113-127.

2. Curtis CF, Otoo LN: A simple model of the build-up of resistance to mixtures of anti-malarial drug. Trans R Soc Trop Med Hyg 1986, 80:889-892.

3. Hastings I: A model for the origins and spread of drug resistant malaria. Parasitology 1997, 115:133-141.

4. Dye C, Williams BG: Multigenic drug resistance among inbred malaria parasites. Proc R Soc Lond B Biol Sci 1997, 264:61-67.

5. Mu JB, Ferdig MT, Feng XR, Joy DA, Duan JH, Furuya T, Subramanian G, Aravind L, Cooper RA, Wootton JC, Xiong M, Su XZ: Multiple transporters associated with malaria parasite responses to chloroquine and quinine. Mol Microbio/ 2003, 49:977-989.

6. Ferdig MT, Cooper RA, Mu JB, Deng BB, Joy DA, Su XZ, Wellems TE: Dissecting the loci of low-level quinine resistance in malaria parasites. Mol Microbiol 2004, 52:985-997.

7. Wang P, Lee C-S, Bayoumi R, Djimde A, Doumbo O, Swedberg G, Dao Le D, Mshinda H, Tanner M, Watkins W, Sims PFG, Hyde JE: Resistance to antifolates in Plasmodium falciparum monitored by sequence analysis of dihydropteroate synthetase and dihydrofolate reductase alleles in a large number of field samples of diverse origins. Mol Biochem Parasitol 1997, 89:161-177.

8. Cowman AF, Morry MJ, Biggs BA, Cross GA, Foote SJ: Amino acid changes linked to pyrimethamine resistance in the dihydrofolate reductase thymidylate synthase gene of Plasmodium falciparum. Proc Natl Acad SCi USA 1998, 85:9109-9113.

9. Peterson DS, Walliker D, Wellems TE: Evidence that a point mutation in dihydrofolate reductase-thymidylate synthase confers resistance to pyrimethamine in falciparum malaria. Proc Natl Acad Sci USA 1988, 85:9114-9118

10. Brooks DR, Wang P, Read M, Watkins WM, Sims PF, Hyde JE: Sequence variation of the hydroxymethyldihydropterin pyrophosphokinase: dihydropteroate synthase gene in lines of the human malaria parasite, Plasmodium falciparum, with differing resistance to sulfadoxine. Eur $J$ Biochem 1994, 224:397-405.
11. Triglia T, Cowman AF: Primary structure and expression of the dihydropteroate synthetase gene of Plasmodium falciparum. Proc Natl Acad Sci USA 1994, 91:7149-7153.

12. Omar SA, Adagu IS, Warhurst DC: Can pretreatment screening for dhps and dhfr point mutations in Plasmodium falciparum infections be used to predict sulfadoxine-pyrimethamine treatment failure? Trans $R S O C$ Trop Med Hyg 2001, 95:315-319.

13. Kublin JK, Dzinjalamala FK, Kamwendo DD, Malkin EM, Cortese JF, Martino LM, Mukadam RAG, Rogerson SJ, Lescano AG, Molyneux ME, Winstanley PA, Chimpeni P, Taylor TE, Plowe CV: Molecular markers for failure of sulfadoxine-pyrimethamine and chlorproguanil-dapsone treatment of Plasmodium falciparum malaria. J Infect Dis 2002, 185:380-388.

14. Steadke SG, Sendagire H, Lamola S, Kamya MR, Dorsey G, Rosenthal PJ: Relationship between age, molecular markers, and response to sulphadoxine-pyrimethamine treatment in Kampala, Uganda. Trop Med Int Health 2004, 9:624-629.

15. Mberu EK, Mosobo MK, Nzila AM, Kokwaro GO, Sibley CH, Watkins WM: The changing in vitro susceptibility pattern to pyrimethamine/ sulfadoxine in Plasmodium falciparum field isolates from Kilifi Kenya. Am J Trop Med Hyg 2000, 62:396-401.

16. Khan B, Omar S, Kanyara JN, Warren-Perry M, Nyalwidhe J, Peterson DS, Wellems T, Kaniaru S, Gitonga J, Mulaa FJ, Koech DK: Antifolate drug resistance and point mutations in Plasmodium falciparum in Kenya. Trans R Soc Trop Med Hyg 1997, 91:456-460.

17. Nzila AM, Nduati E, Mberu EK, Hopkins Sibley C, Monks SA, Winstanley PA, Watkins WM: Molecular evidence of greater selective pressure for drug resistance exerted by the long-acting antifolate pyrimethamine/ sulfadoxine compared with the shorter-acting chlorproguanil/ dapsone on Kenyan Plasmodium falciparum. J Infect Dis 2000 181:2023-2028.

18. Curtis J, Duraisingh MT, Warhurst DC: In vivo selection for a specific genotype of dihydropteroate synthetase of Plasmodium falciparum by pyrimethamine-sufadoxine but not chlorproguanil-dapsone treatment. J Infect Dis 1998, 177:1429-1433.

19. Plowe CV, Cortese JF, Djimde A, Nwanyanwu OC, Watkins WM, Winstanley JG, Estrada-Franco JG, Mollinedo RE, Avila JC, Cespedes JL, Carter D, Doumbo OK: Mutations in Plasmodium falciparum dihydrofolate reductase and dihydropteroate synthase and epidemiologic patterns of pyrimethamine sulfadoxine use and resistance. J Infect Dis 1997, 176:1590-1596.

20. Abdel-Muhsin A, Mackinnon MJ, Ali E, Nassir EA, Suleiman S, Ahmed S, Walliker D, Babiker HA: Evolution of drug-resistance genes in Plasmodium falciparum in an area of seasonal malaria transmission in Eastern Sudan. J Infect Dis 2004, 189:1239-44.

21. Roper C, Pearce R, Bredenkamp B, Gumede J, Drakeley C, Mosha F, Chandramohan $D$, Sharp B: Antifolate antimalarial resistance in southeast Africa. Lancet 2003, 361:1174-81.

22. Mugittu K, Ndejembi M, Malisa A, Lemnge M, Premji Z, Mwita A, Nkya W, Kataraihya J, Abdulla S, Beck H, Mshinda H: Therapeutic efficacy of sulphadoxine-pyrimethamine and prevalence of resistance markers in Tanzania prior to revision of malaria treatment policy: Plasmodium falciparum dihydrofolate reductase and dihydropteroate synthase mutations in monitoring in vivo resistance. Am J Trop Med Hyg 2004, 71:696-702.

23. Ronn AM, Msangeni HA, Mhina J, Wernsdorfer WH, Bygbjerg IC: High level of resistance of Plasmodium falciparum to sulfadoxine-pyrimethamine in children in Tanzania. Trans R Soc Trop Med Hyg 1996, 90:179-181.

24. Trigg JK, Mbwana H, Chambo O, Hills E, Watkins W, Curtis CF: Resistance to pyrimethamine/sulfadoxine in Plasmodium falciparum in 12 villages in north east Tanzania and a test of chlorproguanil/dapsone. Acta Trop 1997, 63:185-189.

25. Schellenberg D, Kahigwa E, Drakeley C, Malende A, Wigayi J, Msokame C, Aponte JJ, Tanner M, Mshinda H, Menendez C, Alonso PL: The safety and efficacy of sulfadoxine-pyrimethamine, amodiaquine and their combination in the treatment of uncomplicated Plasmodium falciparum malaria. Am J Trop Med Hyg 2002, 67:17-23.

26. Pearce R, Drakeley C, Chandramohan D, Mosha F, Roper C: Molecular Determination of Point Mutation Haplotypes in the Dihydrofolate Reductase and Dihydropteroate Synthase of Plasmodium falciparum in Three Districts of Northern Tanzania. Antimicrob Agents Chemother 2003, 47:1347-1354. 
27. Conway DJ, Roper C, Oduola AM, Arnot DE, Kremsner PG, Grobusch MP, Curtis $C F$, Greenwood BM: High recombination rate in natural populations of Plasmodium falciparum. Proc Natl Acad Sci USA 1999, 96:4506-11.

28. StataCorp: Stata Statistical Software: Release 7.0. College Station, TX: Stata Corporation; 2002

29. Bwijo B, Kaneko A, Takechi M, Zungu IL, Moriyama Y, Lum JK, Tsukahara T, Mita T, Takahashi N, Bergqvist Y, Bjorkman A, Kobayakawa T: High prevalence of quintuple mutant $\mathrm{dhfr} / \mathrm{dhps}$ genes in Plasmodium infections seven years after introduction of sufadoxine and pyrimethamine as first line treatment in Malawi. Acta Trop 2003, 85:363-373

30. Nzila AM, Mberu EK, Sulo J, Dayo H, Winstanley PA, Sibley CH, Watkins WM: Towards an understanding of the mechanism of pyrimethaminesulfadoxine resistance in Plasmodium falciparum: Genotyping of dihydrofolate reductase and dihydropteroate synthase of Kenyan parasites. Antimicrob Agents Chemother 2000, 44:991-996.

31. Kyabayinze D, Cattamanchi A, Kamya MR, Rosenthal PJ, Dorsey G: Validation of a simplified method for using molecular markers to predict sulfadoxine-pyrimethamine treatment failure in African children with falciparum malaria. Am J Trop Med Hyg 2003, 69:247-252.

32. Sendagire H, Kyabayinze D, Swedberg G, Kironde F: Plasmodium falciparum: higher incidence of molecular resistance markers for sulphadoxine than for pyrimethamine in Kasangati, Uganda. Trop Med Int Health 2005, 10:537-543.

33. Menard D, Yapou F, Manirakiza A, Djalle D, Matsika-Claquin MD, Talarmin A: Polymorphisms in PFCRT, PFMDR, DHFR genes and in-vitro responses to antimalarials in Plasmodium falciparum isolates from Bangui, Central African Republic. Am J Trop Med Hyg 2006, 75:381-387.

34. Schneider AG, Premji Z, Felger I, Smith T, Abdulla S, Beck H-P, Mshinda H: A point mutation in codon 76 of pfcrt of $P$. falciparum is positively selected for by Chloroquine treatment in Tanzania. Infection, Genetic and Evolution 2002, 1:183-189.

35. Anderson TJ, Nair S, Sudimack D, Williams JT, Mayxay M, Newton PN, JeanPaul Guthmann J, Smithuis FM, Hien TT, van den Broek IVF, White NJ, Nosten F: Geographical distribution of selected and putatively neutral SNPs in Southeast Asian malaria parasites. Mol Biol Evol 2005, 22:2362-2374

36. Enosse S, Magnussen P, Abacassamo F, Gómez-Olivé X, Rønn AM, Thompson R, Alifrangis M: Rapid increase of Plasmodium falciparum $\mathrm{dhfr} / \mathrm{dhps}$ resistant haplotypes, after the adoption of sulphadoxinepyrimethamine as first line treatment in 2002, in southern Mozambique. Malar J 2008, 7:115.

37. Mugittu K, Abdulla S, Falk N, Masanja H, Felger I, Mshinda H, Beck H, Genton B: Efficacy of sulphadoxine-pyrimethamine in Tanzania after two years as first-line drug for uncomplicated malaria: assessment protocol and implication for treatment policy. Malar J 2005, 4:55.

38. Goodman C, Kachur SP, Abdulla S, Mwageni E, Nyoni J, Schellenberg JA, Mills A, Bloland P: Retail supply of malaria-related drugs in rural Tanzania: risks and opportunities. Trop Med Int Health 2004, 9:655-663.

39. Cisse B, Sokhna C, Boulanger D: Seasonal intermittent preventive treatment with artesunate and sulfadoxine-pyrimethamine for prevention of malaria in Senegalese children: a randomised, placebocontrolled, double-blind trial. Lancet 2006, 367:659-67.

40. Marks F, von Kalckreuth V, Kobbe R: Parasitological rebound effect and emergence of pyrimethamine resistance in Plasmodium falciparum after single-dose sulfadoxine-pyrimethamine. J Infect Dis 2005, 192:1962-1965.

41. Pearce R, Malisa A, Kachur SP, Barnes K, Sharp B, Roper C: Reduced Variation Around Drug Resistant dhfr Alleles in African Plasmodium falciparum. Mol Biol Evol 2005, 22:1834-1844.

42. Roper C, Pearce R, Nair S, Sharp B, Nosten F, Anderson T: Intercontinental spread of pyrimethamine resistant malaria. Science 2004, 305:1124.

43. Khalil I, Alifrangis M, Ronn AM, Gabar HA, Jelinek T, Satti GM, Bygbjerg IC: Pyrimethamine/sulfadoxine combination in the treatment of uncomplicated falciparum malaria: relation between dihydropteroate synthase/dihydrofolate reductase genotypes, sulfadoxine plasma levels, and treatment outcom. Am J Trop Med Hyg 2002, 67:225-229.

44. Mendez F, Munoz A, Carrasquilla G, Jurado D, Arevalo-Herrera M, Cortese $J$, Plowe CV: Determinants of treatment response to sulfadoxinepyrimethamine and subsequent transmission potential in falciparum malaria. Am J Epidemiol 2002, 156:230-238.
45. Hastings IM, Watkins WM, White NJ: The evolution of drug-resistant malaria: the role of drug elimination half-life. Philos Trans $R$ Soc London Ser B 2002, 357:505-519.

46. Clyde D: Malaria in Tanzania. Oxford: Oxford University Press; 1967

47. Avery-Jones S: Mass treatment with pyrimethamine. A study of resistance and cross resistance resulting from a field trial in the hyperendemic malarious area of Makueni, Kenya Sept 1952-Sept 1953. Trans R Soc Trop Med Hyg 1958, 52:547-561.

48. Peters W: Chemotherapy and Drug Resistance in Malaria. London: Academic Press; 1970.

49. Nzila-Mounda A, Mberu EK, Sibley CH, Plowe CV, Winstanley P: Kenyan Plasmodium falciparum field isolates: correlation between pyrimethamine and chlorcycloguanil activity in vitro and point mutations in the dihydrofolate reductase domain. Antimicrob Agents Chemother 1998, 42:164-168.

50. Certain LK, Briceno M, Kiara SM, Nzila AM, Watkins WM, Sibley CH: Characteristics of Plasmodium falciparum dhfr haplotypes that confer pyrimethamine resistance, Kilifi, Kenya, 1987-2006. J Infect Dis 2008, 197:1743-51.

51. Talisuna AO, Okelo PE, Erhart A, Coosemans M, D'Allessandro U: Intensity of malaria transmission and the spread of Plasmodium falciparum Resistant malaria: A review of epidemiologic field evidence. Am J Trop Med Hyg 2007, 77:170-180

52. Mackinnon $\mathrm{MJ}$, Hastings $\mathrm{IM}$ : The evolution of multiple drug resistance in malaria parasites. Trans R Soc Trop Med Hyg 1998, 92:188-195.

53. Hastings IM: Complex dynamics and stability of antimalarial drug resistance. Parasitol 2006, 132:615-624.

54. Noranate N, Durand R, Tall A, Marrama L, Spiegel A, Sokhna C, Pradines B, Cojean S, Guillotte M, Bischoff E, Ekala M, Bouchier C, Fandeur T, Ariey F, Patarapotikul J, Le Bras J, Trape J, Rogier C, Mercereau-Puijalon O: Rapid dissemination of Plasmodium falciparum drug resistance despite strictly controlled antimalarial use. PloS ONE 2007:e139.

doi: $10.1186 / 1475-2875-9-190$

Cite this article as: Malisa et al., Drug coverage in treatment of malaria and the consequences for resistance evolution - evidence from the use of sulphadoxine/pyrimethamine Malaria Journal 2010, 9:190

\section{Submit your next manuscript to BioMed Central and take full advantage of:}

- Convenient online submission

- Thorough peer review

- No space constraints or color figure charges

- Immediate publication on acceptance

- Inclusion in PubMed, CAS, Scopus and Google Scholar

- Research which is freely available for redistribution 